Review Article

\title{
Epigenetic Regulation of Hepatocellular Carcinoma Progression through the mTOR Signaling Pathway
}

\author{
Mengnan Guo ${ }^{D},{ }^{1}$ Ning Li, ${ }^{2}$ Jianxia Zheng, ${ }^{1}$ Wei Wang, ${ }^{1}$ Yan $\mathrm{Wu}^{2}{ }^{2}$ Xu Han, ${ }^{2}$ Jiapei Guo, ${ }^{2}$ \\ Weixi Chen, ${ }^{2}$ Zekun Bai, ${ }^{2}$ Wen Bai, ${ }^{2}$ and Jinghua $W u{ }^{1}{ }^{1}$ \\ ${ }^{1}$ Tangshan Maternal and Child Health Care Hospital, North China University of Science and Technology, Tangshan 063000, \\ Hebei, China \\ ${ }^{2}$ North China University of Science and Technology Affiliated Hospital, Tangshan 063000, Hebei, China
}

Correspondence should be addressed to Jinghua Wu; tswujinghua@163.com

Received 21 February 2021; Accepted 11 May 2021; Published 25 May 2021

Academic Editor: José L. Mauriz

Copyright ( 92021 Mengnan Guo et al. This is an open access article distributed under the Creative Commons Attribution License, which permits unrestricted use, distribution, and reproduction in any medium, provided the original work is properly cited.

Hepatocellular carcinoma (HCC), the most common type of primary liver cancer, is an aggressive tumor with a high mortality rate because of the limited systemic and locoregional treatment modalities. The development and progression of HCC depend on epigenetic changes that result in the activation or inhibition of some signaling pathways. The mTOR signaling pathway is essential for many pathophysiological processes and is considered a major regulator of cancer. Increasing evidence has shown that epigenetics plays a key role in HCC biology by regulating the mTOR signaling pathway. Therefore, epigenetic regulation through the mTOR signaling pathway to diagnose and treat HCC will become a very promising strategy.

\section{Introduction}

Hepatocellular carcinoma (HCC) is the fifth most common malignancy in men and the seventh most common malignancy in women worldwide and results in more than 700,000 deaths each year [1-3]. Further deterioration of HCC can be prompted by failure of early diagnosis, lack of effective therapeutic targets, and inadequate surveillance. Therefore, the development of novel diagnostic methods and the identification of novel targets for therapeutic intervention are urgently needed for the diagnosis and treatment of HCC [4].

Epigenetics is a heritable phenomenon that affects gene expression without altering the DNA sequence [5]. Epigenetics has been found to regulate gene expression mainly through four mechanisms: DNA methylation, histone modification, chromatin remodeling, and noncoding RNA regulation [6,7]. Aberrant epigenetic regulation plays an important role in the development of human malignancies, including HCC. Therefore, better understanding of the abnormal epigenetic regulations in HCC may provide new options for the diagnosis and treatment of HCC. In addition, epigenetics also regulates signaling pathways such as the NF$\kappa \mathrm{B}, \mathrm{RAF} / \mathrm{MEK} / \mathrm{ERK}$, JAK-STAT, Wnt, Notch, and MAPK as well as mTOR pathways [8-10]. Moreover, alterations in these signaling pathways can lead to changes in the biological traits of HCC cells to some extent [11]. Among these signaling pathways, the mTOR signaling pathway plays an important role in affecting HCC progression.

mTOR is involved in multiple signaling pathways that regulate cell proliferation, autophagy, and apoptosis in vivo [12]. There are two main mTOR signaling pathways: the classical PI3K/Akt/mTOR signaling pathway and the LKB1/ AMPK/mTOR signaling pathway. Mutation, activation, and silencing of $\mathrm{mTOR}$ upstream genes, thereby regulating the mTOR pathway, affect the development process of HCC [7]. In addition, mTOR can also regulate protein synthesis by phosphorylating downstream target proteins p70s6k kinase (e. g., 6K1 and 4EBP1), which then regulate mRNA translation [13]. Therefore, changes in upstream and downstream genes of mTOR leading to dysregulation of mTOR signaling pathway will affect the progression of HCC. More than 70\% 
of cancers are known to result in hyperactivation of mTOR. mTOR inhibitors have thus been widely studied for their use in cancer treatment, and some of these inhibitors (e. g., rapamycin, everolimus, doxorubicin, and sorafenib) are used for the treatment of HCC. However, clinical studies have shown that mTOR inhibitors have some limitations, such as low bioavailability and toxicity, and some cancers eventually acquire drug resistance [14-16]. Based on the limited efficacy of mTOR inhibitors, other novel drugs targeting mTOR need to be identified. Several mTOR inhibitors are currently under investigation for the treatment of HCC, and although many preclinical and clinical trial studies have been conducted, these inhibitors have not been applied. Therefore, the discovery of novel regulators of mTOR may become a new therapeutic target for HCC.

In a review, we systematically summarize information regarding the regulation of the mTOR signaling pathway by factors mutated in epigenetic inheritance for the diagnosis and treatment of HCC (Figure 1).

\section{2. ncRNAs}

Noncoding RNA (ncRNA) refers to RNA that does not encode proteins [17]. Advances in sequencing technologies have led to the discovery of many ncRNA species, some of which are highly conserved, such as microRNAs (miRNAs), ultraconserved regions of transcription [18] and circRNAs (circRNAs), as well as ncRNAs that are not conserved between other species, such as long noncoding RNAs (lncRNAs) [19]. Studies have demonstrated that ncRNAs regulate cellular processes and pathways in developmental and pathological contexts [20]. In human diseases, particularly cancer, deregulated expression of ncRNAs can lead to changes in signaling pathways that can affect tumor development. Research has shown that ncRNAs are key players in human carcinogenesis and therefore may play potential roles in the diagnosis and treatment of cancer.

2.1. MiRNAs and HCC. MiRNAs are a diverse family of highly conserved ncRNAs with sizes that range from approximately 18 to 25 nucleotides. MiRNAs pair by complete or incomplete base complementation with the $3^{\prime}$ untranslated region of mRNA. This pairing leads to the degradation or inhibited translation of the target mRNA, with subsequent effects on protein expression. MiRNAs participate in the processes of cell growth, differentiation, development, proliferation, apoptosis, and metabolism [21, 22]. MiRNAs also play an important role in the physiological and pathological processes of cancer, including HCC. In recent years, a large number of studies have shown that the expression levels of miRNAs in HCC are upregulated or downregulated to varying degrees, suggesting that they play a role in various biological processes such as growth, proliferation, and apoptosis of HCC cells [23-26]. Therefore, miRNAs are very promising targets in the diagnosis and treatment of HCC.

2.1.1. MiRNA Regulation of the PI3K/AKT/mTOR Signaling Pathway in HCC. HCC is the third leading cause of cancer- related death worldwide, and its incidence continues to rise. Although cirrhosis underlies most cases of HCC, many molecular pathways are closely associated with HCC carcinogenesis [27]. For example, activation or upregulation of the PI3K/AKT/mTOR signaling pathway can affect the occurrence and development of tumors [28]. The deregulation of this pathway has been shown to result from the deregulation of miRNAs. In HCC induced by mTOR signaling, the expressions of some miRNAs are decreased, and upregulation of these miRNAs is required to inhibit HCC development, while some miRNAs are increased in HCC (Table 1). A total of sixteen miRNAs (miR-1914 [29], miR192-5p [30], miR-486-5p [31], miR-601 [32], miR-132 [33], miR-1207-5p [34], miRNA-133b [35], miR-144-3p [36], miR26a [37], miR-21 [38], miR-199a-3p [39, 40], miR-758-3p [41], miR-494 [42], miR-125a [43], miR-345 [44], and miR29a-3p [45]) have been identified as downregulated in HCC. Three miRNAs (miR-9-5p [46], miR-300 [47], and miR-181a [48]) were identified as upregulated in HCC.

2.1.2. MiRNA Regulation of Other $m$ TOR Signaling Pathways in HCC. In addition to miRNA-mediated regulation of the classical PI3K/AKT/mTOR signaling pathway, miRNAs can also affect HCC development through other mTOR signaling pathways.

In HCC, upregulation of miR-18a and miR-25 is associated with poor patient survival and promotion of HCC cell line proliferation. Sanchez-Mejias et al. [49] analyzed the predicted targets of some miRNAs and validated SOCS5 as a bonafide target of miR-18a and miR-25. Furthermore, the authors demonstrated that the SOCS5/miR-18a/miR-25 axis inhibits HCC development by regulating downstream mTOR signaling.

Zhou et al. [50] found that miR-100 downregulation was closely related to the development of HC. MiR-100 reduces the protein level of angiopoietin 2 (Angpt2) by targeting mTOR and blocking the mTOR-p70S6K signaling pathway, which in turn inhibits the formation of encapsulated tumor clusters (VETC), thereby eliminating VETC-dependent metastasis of HCC cells. These results indicate miR-100 as a new target for antimetastatic therapy of HCC.

Meanwhile, the experimental results of Dong et al. [51] showed that miR-223 could inhibit cell growth and promote apoptosis in HepG2 and Bel-7402 hepatoma cell lines and screened a novel miR-223 target, the Ras-related protein Rab-1 (Rab1). MiR-223 mediates mTOR signaling pathway inactivation by targeting Rab1, thereby inhibiting tumorigenesis and promoting HCC apoptosis. Therefore, miR-223 could be a potential therapeutic target for the treatment of HCC.

These studies indicate miRNAs can affect the progression of liver cancer by regulating the mTOR signaling pathway and provide a new approach for the diagnosis and treatment of liver cancer.

2.2. LncRNAs and HCC. Among the various types of ncRNAs, lncRNAs have received increasing attention. LncRNAs are defined as transcripts of more than 200 


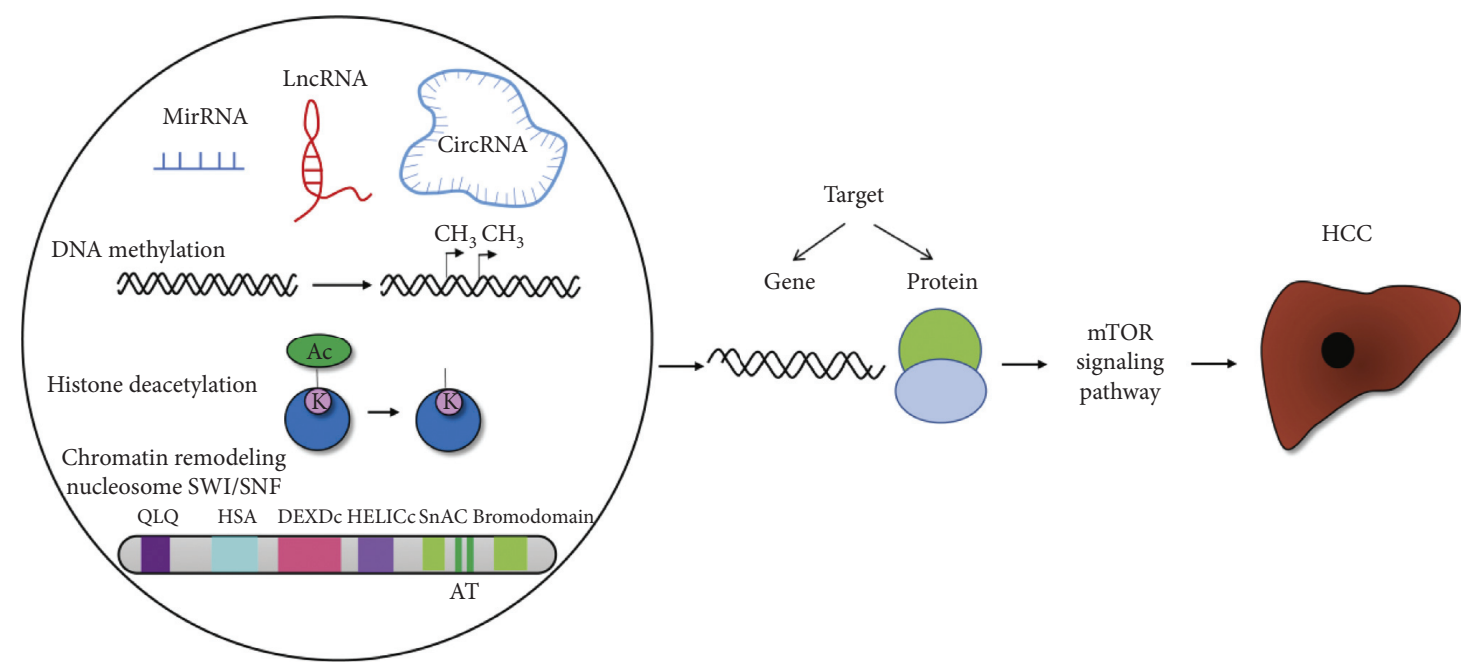

FIGURE 1: Epigenetic regulation of the mTOR signaling pathway in HCC.

TABle 1: Dysregulated miRNAs in HCC.

\begin{tabular}{lccc}
\hline & MiRNAs & Target & Expression \\
\hline Sun et al. & MiR-1914 & GPR39 & Downregulated \\
Zhu et al. & MiR-192-5p & TRIP13 & Downregulated \\
Youness et al. & MiR-486-5p & IGF-1R & Downregulated \\
Song et al., Liu et al. & MiR-601, miR-132 & PIK3R3 & Downregulated \\
Zhao et al. & MiR-1207-5p & FASN & Downregulated \\
Wang et al. & MiR-133b & EGFR & Downregulated \\
Wu et al. & MiR-144-3p & SGK3 & Downregulated \\
Sun et al. & MiR-26a & ST3GAL & Downregulated \\
Xia et al. & MiR-21 & PTEN & Downregulated \\
Callegari et al., Lou G et al. & MiR-199a-3p & mTOR pathway & Downregulated \\
Jiang et al. & MiR-758-3p & mTOR pathway & Downregulated \\
Pollutri et al. & MiR-494 & mTOR pathway & Downregulated \\
Tang et al. & MiR-125a & mTOR pathway & Downregulated \\
Yu et al. & MiR-345 & [RF1 & Downregulated \\
Song et al. & MiR-29a-3p & [39, 40] \\
Dong et al. & MiR-9-5p & Robo1 & Downregulated \\
Chang et al. & MiR-300 & KLF4 & Upregulated \\
Chang et al. & MiR-181a & FOXO1 & Upregulated \\
\hline
\end{tabular}

nucleotides that lack protein-encoding ability [52]. LncRNAs regulate the expression of genes by epigenetic regulation, transcriptional regulation, and posttranscriptional regulation in the form of RNA [53]. Therefore, $\operatorname{lncRNAs}$ have been shown to function as master regulators of gene expression and can play critical roles in various biological functions and disease processes in cancer, including HCC [54]. The genome-wide expression pattern of lncRNAs in HCC cells or tissues and their tissue-specific expression characteristics have been found by a number of studies, and lncRNAs hold promise as novel diagnostic biomarkers and therapeutic targets for HCC [55].

2.2.1. LncRNAs Regulate the PI3K/Akt/mTOR Signaling Pathway in HCC. With the development of next-generation sequencing, more long noncoding RNAs (lncRNAs) were found. Initially, lncRNAs were considered as "noisy" transcripts or "dark matter" [56]. An increasing number of studies have revealed the indispensable role of lncRNAs in the dysregulation of signaling pathways in HCC [57]. The impact of lncRNAs on the progression of HCC through the $\mathrm{PI} 3 \mathrm{~K} / \mathrm{Akt} / \mathrm{mTOR}$ signaling pathway is relatively novel. Upregulation or downregulation of $\mathrm{PI} 3 \mathrm{~K} / \mathrm{AKT} / \mathrm{mTOR}$-related oncogenic lncRNAs contributes to aberrant expression of transcriptional activators or oncoproteins, leading to aberrant regulation of the $\mathrm{PI} 3 \mathrm{~K} / \mathrm{AKT} / \mathrm{mTOR}$ pathway in HCC [58]. In addition, lncRNAs are aberrantly expressed in liver cancer and are significantly associated with metastasis, recurrence, prognosis, and chemoresistance of HCC [59]. Here, we summarize that 13 lncRNAs are dysregulated in HCC through the PI3K/Akt/mTOR signaling pathway. Twelve of the lncRNAs (lncRNA DCST1-AS1 [60], lncRNA OGFRP1 [61], lncRNA TMPO-AS1 [62], lncRNA MALAT1 
[63, 64], lncRNA AK023948 [65], IncRNA HAGLROS [66], lncRNA CDKN2B-AS1 [67], IncRNA SNHG16 [68], lncRNA CASC11 [69], IncRNA DUXAP10 [70], IncRNA PICSAR [71], and IncRNA LINC00680 [72]) were upregulated in HCC, while lncRNA HULC [73] was downregulated in HCC (Table 2).

\subsubsection{LncRNAs Modulate the Role of Other mTOR Signaling} Pathways in HCC. Several studies have shown that lncRNAs also regulate other mTOR signaling pathways in liver cancer. $\mathrm{Ma}$ and colleagues [74] found that abnormal expression of the lncRNA HEIH was associated with HCC cell growth and metastasis. HEIH was highly expressed in tumor tissue, and HEIH reduction significantly reduced Huh7 and Hep3B hepatoma cell viability, migration, and invasion and induced apoptosis. MiR-199a-3p was identified as a downstream effector of HEIH, and the functional effects of HEIH on Huh7 and Hep3B cells were attenuated when the miR-199a$3 p$ expression was inhibited. In addition, reduced HEIH inhibited the activation of mTOR signaling by upregulating miR-199a-3p, indicating that HEIH may be a potential target for HCC.

Wei and colleagues [75] found significantly increased HOTAIR expression in 84 HCC tissues compared with nontumor tissues and determined the effect of HOTAIR on HCC cell-regulated glucose metabolism by examining lactate and glucose levels. The authors found that HOTAIR promotes glycolysis by upregulating glucose transporter isoform 1 (GLUT1) and activating mTOR signaling, while downregulation of HOTAIR inhibits this effect. This study revealed a new relationship between HOTAIR and glucose metabolism in HCC cells and indicated HOTAIR as a new target for the diagnosis and treatment of HCC.

Li et al. [76] found for the first time the upregulation of lncRNA-OR3A4 in HCC tissues and cell lines and identified OR3A4 as a promoter of HCC progression and angiogenesis. OR3A4 regulates HCC proliferation, metastasis, and angiogenesis through AGGF1/AKT/mTOR. The authors revealed that OR3A4 is a novel predicted target for HCC.

The lncRNA HIF1A-AS1 is overexpressed in HCC tissues and is associated with tumor size, TNM stage, and lymph node metastasis. Hong et al. [77] found that HIF1AAS1 promotes hepatocarcinogenesis by activating autophagy through the HIF- $1 \alpha /$ mTOR signaling pathway. The authors showed that HIF1A-AS1 is involved in regulating the progression of HCC and provided a potential direction for future HCC treatment strategies.

These reports indicate that dysregulation of IncRNAs can activate the mTOR signaling pathway to regulate the development of HCC, taking the understanding and potential use of lncRNAs in the diagnosis and treatment of liver cancer patients to a new level.

2.3. CircRNAs and HCC. CircRNAs are a novel class of ncRNAs characterized by a covalent closed-loop structure without a $5^{\prime}$ cap structure or a $3^{\prime}$ polyA tail [78]. Since the first discovery in viruses in the 1970s [79], circRNAs have begun to attract much attention, and research has been focused on their biogenesis, characteristics, functional mechanisms, and potential applications in clinical diagnosis and treatment. CircRNAs regulate gene expression at the transcriptional, posttranscriptional, and translational levels; they also regulate alternative splicing, sponge miRNAs, and sequester functional proteins. CircRNAs are involved in many pathological processes such as Alzheimer's disease, diabetes, atherosclerosis, and glioma [80-83]. CircRNAs play an important role in cancer growth, metastasis, recurrence, and treatment resistance [84].

The relationship between circRNAs and HCC has become a research hotspot in the past two years. CircRNAs were shown to have important regulatory roles in HCC development, and progression. In HCC, some circRNAs act as oncogenes and can promote the proliferation and $\mathrm{mi}-$ gration of cancer, while other circRNAs act as tumor suppressors and can induce apoptosis of HCC cells. Although some studies have demonstrated that circRNAs can affect the progression of HCC, the roles of circRNAs on HCC remain largely unknown [85]. Therefore, the functions and mechanisms of circRNAs in HCC need to be further studied.

\subsubsection{CircRNAs Modulate the mTOR Signaling Pathway in} HCC. CircRNA can affect the development and progression of HCC through the mTOR signaling pathway [86]. For example, Huang et al. [87] showed that circRNA-100338 activation of the mTOR signaling pathway through the circRNA-100338/miR-141-3p/RHEB axis is closely related to the poor prognosis of hepatitis B-related HCC. This study makes the connection between circRNA-100338 and mTOR signaling pathway in HCC cells and may provide a potential therapeutic target for HCC.

Sun et al. [88] found that three circRNAs-circRNA0004001, circRNA 0004123, and circRNA0075792-were upregulated in HCC blood samples using qRT-PCR. The expression of the three circRNAs was positively correlated with TNM stage and tumor size, and the three circRNA combination targeted a variety of miRNAs to participate in the mTOR signaling pathway, correlated with the development of liver cancer, and may be valuable diagnostic biomarker for HCC.

Zheng et al. [89] found that hsa-circ-0079929 was expressed at low levels in HCC. CircRNA-0079299 overexpression inhibited HCC growth and delayed cell cycle progression in vitro and in vivo but had no effect on cell migration and apoptosis. The inhibition of HCC growth by circRNA-0079299 is mediated by the $\mathrm{PI} 3 \mathrm{~K} / \mathrm{AKT} / \mathrm{mTOR}$ signaling pathway.

Although few reports have shown that circRNAs play a role in HCC through the mTOR signaling pathway, future studies clarifying their role in HCC development may lead to new approaches for the diagnosis and treatment of HCC.

\section{DNA Methylation}

DNA methylation is an important epigenetic modification that occurs mainly at the CpG islands of DNA and involves either hypermethylation or hypomethylation. Aberrant 
TABle 2: Dysregulated lncRNAs in HCC.

\begin{tabular}{|c|c|c|c|c|}
\hline & LncRNA & Target & Expression & Reference \\
\hline Li et al. & LncRNA DCST1-AS1 & mTOR pathway & Upregulated & {$[60]$} \\
\hline Chen et al. & LncRNA OGFRP1 & mTOR pathway & Upregulated & {$[61]$} \\
\hline Guo et al. & LncRNA TMPO-AS1 & FOXK1 & Upregulated & {$[62]$} \\
\hline Peng et al. & LncRNA MALAT1 & PI3k3' noncoding region & Upregulated & {$[63]$} \\
\hline Malakar et al. & LncRNA MALAT1 & TCF7L2 & Upregulated & {$[64]$} \\
\hline Ye et al. & LncRNA AK023948 & mTOR pathway & Upregulated & {$[65]$} \\
\hline Wei et al. & LncRNA HAGLROS & MiR-5059/AGLROS axis & Upregulated & [66] \\
\hline Zheng et al. & LncRNA CDKN2B-AS1 & Let-7c-5p/NAP1L1 axis & Upregulated & [67] \\
\hline Zhong et al. & LncRNA SNHG16 & p62 & Upregulated & {$[68]$} \\
\hline Han et al. & LncRNA CASC11 & PTEN & Upregulated & [69] \\
\hline Sun et al. & LncRNA DUXAP10 & GRP39 & Upregulated & {$[70]$} \\
\hline Liu et al. & LncRNA PICSAR & MiR-588 & Upregulated & {$[71]$} \\
\hline Shu et al. & LncRNA LINC00680 & AKT3 & Upregulated & {$[72]$} \\
\hline Xin et al. & LncRNA HULC & PTEN & Downregulated & {$[73]$} \\
\hline
\end{tabular}

DNA methylation in cancer has been heralded as a promising target for the development of powerful diagnostic, prognostic, and predictive biomarkers [90]. For example, Abeni et al. [10] found that oncogene hypermethylation led to activation of mTOR signaling and inhibited tumor progression after sorafenib treatment. In addition, Liu et al. [91] found that the BCLB gene is methylated in HCC. Hypermethylated BCLB, which induces both apoptosis and autophagy in HCC cells through the AMPK-mTOR signaling cascade, plays a role in cancer suppression and has therapeutic implications for HCC patients.

3.1. Histone Modification. Histone modifications, as a class of epigenetic regulatory mechanisms that regulate gene expression, have received increasing attention because their modification pattern changes are closely related to the development of a variety of malignancies [92]. For example, cell differentiation and organismal development and abnormal modifications of histones contribute to diseases such as cancer [93]. Zhang et al. [94] found that acetazolamide (SIRT1), a NAD+-dependent histone deacetylase, exerts antioncogenic effects in HCC through the AMPK-mTOR pathway in the context of mutant p53. Wang et al. [95] found that inhibition of histone methyltransferase 3 (SMYD3) resulted in reduced $\mathrm{AKT} / \mathrm{mTOR}$ signaling activity, which triggered deleterious effects on bladder cancer cells. In addition, Makarević et al. [96] found that decreased acetylation of $\mathrm{H} 3$ and $\mathrm{H} 4$ promotes prostate cancer cell development by activating the mTOR signaling pathway in prostate cancer. Sun et al. [97] found that inhibition of mTOR signaling enhanced trichostatin A and promoted histone acetylation in gastric cancer cell lines. To date, there have been no reports on the role of histone methylation and acetylation in liver cancer through the mTOR signaling pathway.

\section{Chromatin Remodeling}

The SWI/SNF complex, originally discovered in yeast 20 years ago, is a family of multi-subunit complexes that use the energy of ATP hydrolysis to remodel nucleosomes.
Chromatin remodeling processes mediated by the SWI/SNF complex are essential for the regulation of gene expression in a variety of cellular processes, including differentiation and proliferation [98]. Many studies have found that the chromatin SWI/SNF complex plays an important role in malignant tumors. Zhou and his team [99] discovered SMARCD1, a subunit of the SWI/SNF complex, as a promising prognostic predictor that promotes liver cancer growth through the mTOR pathway.

\section{Conclusions and Perspectives}

Multiple studies have demonstrated that epigenetic changes play an important role in the development and progression of HCC. The mTOR pathway is involved in the growth and proliferation of HCC cells, and epigenetic regulation through mTOR signaling will affect HCC progression. As supported by increasing evidence, epigenetic regulators through the mTOR signaling pathway as ideal therapeutic targets for HCC are a potent future research direction. Although there have been major breakthrough in epigenetics in the treatment of HCC, some questions remain unanswered. For example, studies linking histone methylation and acetylation with the mTOR signaling pathway are scarce. Future studies should pursue this research direction to expand the exploration of strategies for HCC treatment targeting the mTOR pathway.

\section{Data Availability}

The data used to support this study are included within this article.

\section{Conflicts of Interest}

The authors declare that they have no conflicts of interest.

\section{Authors' Contributions}

All authors made substantial contributions to acquisition of data, or analysis, conception and design, and interpretation of data; took part in drafting of the article or revising it critically for important intellectual content; gave final 
approval of the version to be published; and agreed to be accountable for all aspects of the work. All authors have read and agreed to the published version of the manuscript.

\section{Acknowledgments}

The authors thank Gabrielle White Wolf, Ph.D., from Liwen Bianji, Edanz Editing China (http://www.liwenbianji.cn), for editing the English text of a draft of this manuscript. This study was supported by grants from the Natural Science Foundation of Hebei Province (grant no. H2019209355), the Science and Technology Research Project of Hebei Higher Education Institutions (grant no. ZD2018090), the Hebei Provincial Health Commission Office (grant no. G2019074), the High-End Talent Funding Project in Hebei Province (grant no. A202003005), and Project of Hebei Provincial Department of Education (grant no. KCJSZ2020056).

\section{References}

[1] P. Ferenci, M. Fried, D. Labrecque et al., "Hepatocellular carcinoma (HCC)," Journal of Clinical Gastroenterology, vol. 44, no. 4, pp. 239-245, 2010.

[2] K. Schütte, J. Bornschein, and P. Malfertheiner, "Hepatocellular carcinoma--epidemiological trends and risk factors," Digestive Diseases, vol. 27, no. 2, pp. 80-92, 2009.

[3] L. A. Torre, "Global cancer statistics," CA: A Cancer Journal for Clinicians, vol. 65, no. 2, pp. 87-108, 2012.

[4] Y.-L. Zheng, L. Li, Y.-X. Jia et al., "LINC01554-Mediated glucose metabolism reprogramming suppresses tumorigenicity in hepatocellular carcinoma via downregulating PKM2 expression and inhibiting akt/mTOR signaling pathway," Theranostics, vol. 9, no. 3, pp. 796-810, 2019.

[5] J. Gayon, "From Mendel to epigenetics: history of genetics," Comptes Rendus Biologies, vol. 339, no. 7-8, pp. 225-230, 2016.

[6] J. S. Ramirez-Prado, A. A. Abulfaraj, N. Rayapuram, M. Benhamed, and H. Hirt, "Plant immunity: from signaling to epigenetic control of defense," Trends in Plant Science, vol. 23, no. 9, pp. 833-844, 2018.

[7] T. Ersahin, N. Tuncbag, and R. Cetin-Atalay, "The PI3K/AKT/ mTOR interactive pathway," Molecular BioSystems, vol. 11, no. 7, pp. 1946-1954, 2015.

[8] J. Torresi, "HBV-related hepatocarcinogenesis: the role of signalling pathways and innovative ex vivo research models," BMC Cancer, vol. 19, no. 1, p. 707, 2019.

[9] K. Song, J. Wu, and C. Jiang, "Dysregulation of signaling pathways and putative biomarkers in liver cancer stem cells (Review)," Oncology Reports, vol. 29, no. 1, pp. 3-12, 2013.

[10] E. Abeni, A. Salvi, E. Marchina, M. Traversa, B. Arici, and G. De Petro, "Sorafenib induces variations of the DNA methylome in HA22T/VGH human hepatocellular carcinoma-derived cells," International Journal of Oncology, vol. 51, no. 1, pp. 128-144, 2017.

[11] P. A. Farazi and R. A. DePinho, "Hepatocellular carcinoma pathogenesis: from genes to environment," Nature Reviews Cancer, vol. 6, no. 9, pp. 674-687, 2006.

[12] Z. Zou, "mTOR signaling pathway and mTOR inhibitors in cancer: progress and challenges," Cell \& Bioscience, vol. 10, p. 31, 2020.

[13] D. Tewari, P. Patni, A. Bishayee, A. N. Sah, and A. Bishayee, "Natural products targeting the PI3K-Akt-mTOR signaling pathway in cancer: a novel therapeutic strategy," Seminars in Cancer Biology, vol. 19, 2019.
[14] M. Dimri and A. Satyanarayana, "Molecular signaling pathways and therapeutic targets in hepatocellular carcinoma," Cancers (Basel), vol. 12, no. 2, 2020.

[15] S. G. Swamy, V. H. Kameshwar, P. B. Shubha et al., "Targeting multiple oncogenic pathways for the treatment of hepatocellular carcinoma," Targeted Oncology, vol. 12, no. 1, pp. 1-10, 2017.

[16] F. Janku, T. A. Yap, and F. Meric-Bernstam, "Targeting the PI3K pathway in cancer: are we making headway?" Nature Reviews Clinical Oncology, vol. 15, no. 5, pp. 273-291, 2018.

[17] M. Matsui and D. R. Corey, "Non-coding RNAs as drug targets," Nature Reviews Drug Discovery, vol. 16, no. 3, pp. 167-179, 2017.

[18] G. Bejerano, "Ultraconserved elements in the human genome," Science, vol. 304, no. 5675, pp. 1321-1325, 2004.

[19] B. Aryal and Y. Suárez, "Non-coding RNA regulation of endothelial and macrophage functions during atherosclerosis," Vascular Pharmacology, vol. 114, pp. 64-75, 2019.

[20] E. Anastasiadou, L. S. Jacob, and F. J. Slack, "Non-coding RNA networks in cancer," Nature Reviews Cancer, vol. 18, no. 1, pp. 5-18, 2018.

[21] W. X. Xu, Z Liu, F Deng et al., "MiR-145: a potential biomarker of cancer migration and invasion," American Journal of Translational Research, vol. 11, no. 11, pp. 6739-6753, 2019.

[22] R. Rupaimoole and F. J. Slack, "MicroRNA therapeutics: towards a new era for the management of cancer and other diseases," Nature Reviews Drug Discovery, vol. 16, no. 3, pp. 203-222, 2017.

[23] J. Brennecke, D. R Hipfner, A Stark, R. B Russell, and S. M Cohen, "Bantam encodes a developmentally regulated microRNA that controls cell proliferation and regulates the proapoptotic gene hid in Drosophila," Cell, vol. 113, no. 1, pp. 25-36, 2003.

[24] P. Xu, S. Y. Vernooy, M. Guo, and B. A. Hay, "The Drosophila microRNA Mir-14 suppresses cell death and is required for normal fat metabolism," Current Biology, vol. 13, no. 9, pp. 790-795, 2003.

[25] A. Lujambio and S. W. Lowe, "The microcosmos of cancer," Nature, vol. 482, no. 7385, pp. 347-355, 2012.

[26] S. Bandiera, S. Pfeffer, T. F. Baumert, and M. B. Zeisel, "miR122-a key factor and therapeutic target in liver disease," Journal of Hepatology, vol. 62, no. 2, pp. 448-457, 2015.

[27] T. Couri and A. Pillai, "Goals and targets for personalized therapy for HCC," Hepatology International, vol. 13, no. 2, pp. 125-137, 2019.

[28] A. Guerrero-Zotano, I. A. Mayer, and C. L. Arteaga, "PI3K/ $\mathrm{AKT} / \mathrm{mTOR}$ : role in breast cancer progression, drug resistance, and treatment," Cancer and Metastasis Reviews, vol. 35, no. 4, pp. 515-524, 2016.

[29] L. Sun, L. Wang, T. Chen et al., "microRNA-1914, which is regulated by lncRNA DUXAP10, inhibits cell proliferation by targeting the GPR39-mediated PI3K/AKT/mTOR pathway in HCC," Journal of Cellular and Molecular Medicine, vol. 23, no. 12, pp. 8292-8304, 2019.

[30] M. X. Zhu, "Elevated TRIP13 drives the AKT/mTOR pathway to induce the progression of hepatocellular carcinoma via interacting with ACTN4," Journal of Experimental \& Clinical Cancer Research, vol. 38, no. 1, p. 409, 2019.

[31] R. A. Youness, H. M. El-Tayebi, R. A. Assal, K. Hosny, G. Esmat, and A. I. Abdelaziz, "MicroRNA-486-5p enhances hepatocellular carcinoma tumor suppression through repression of IGF-1R and its downstream mTOR, STAT3 and c-Myc," Oncology Letters, vol. 12, no. 4, pp. 2567-2573, 2016. 
[32] Y. Song, "MicroRNA-601 serves as a potential tumor suppressor in hepatocellular carcinoma by directly targeting PIK3R3," Molecular Medicine Reports, vol. 19, no. 3, pp. 2431-2439, 2019.

[33] K. Liu, X. Li, Y. Cao, Y. Ge, J. Wang, and B. Shi, "MiR-132 inhibits cell proliferation, invasion and migration of hepatocellular carcinoma by targeting PIK3R3," International Journal of Oncology, vol. 47, no. 4, pp. 1585-1593, 2015.

[34] G. Zhao, L. Dong, H. Shi et al., "MicroRNA-1207-5p inhibits hepatocellular carcinoma cell growth and invasion through the fatty acid synthase-mediated Akt/mTOR signalling pathway," Oncology Reports, vol. 36, no. 3, pp. 1709-1716, 2016.

[35] X. Wang, J. Zeng, L. Wang et al., "Overexpression of microRNA-133b is associated with the increased survival of patients with hepatocellular carcinoma after curative hepatectomy: involvement of the EGFR/PI3K/Akt/mTOR signaling pathway," Oncology Reports, vol. 38, no. 1, pp. 141-150, 2017.

[36] M. Wu, C. Huang, X. Huang, R. Liang, Y. Feng, and X. Luo, "MicroRNA-144-3p suppresses tumor growth and angiogenesis by targeting SGK3 in hepatocellular carcinoma," Oncology Reports, vol. 38, no. 4, pp. 2173-2181, 2017.

[37] M. Sun, X. Zhao, L. Liang, X. Pan, H. Lv, and Y. Zhao, "Sialyltransferase ST3GAL6 mediates the effect of microRNA$26 \mathrm{a}$ on cell growth, migration, and invasion in hepatocellular carcinoma through the protein kinase $\mathrm{B} /$ mammalian target of rapamycin pathway," Cancer Science, vol. 108, no. 2, pp. 267-276, 2017.

[38] C. Xia, H. Zeng, and Y. Zheng, "Low-intensity ultrasound enhances the antitumor effects of doxorubicin on hepatocellular carcinoma cells through the ROS-miR-21-PTEN axis," Molecular Medicine Reports, vol. 21, no. 3, pp. 989-998, 2020.

[39] E. Callegari, L. D’Abundo, P. Guerriero et al., "miR-199a-3p modulates MTOR and PAK4 pathways and inhibits tumor growth in a hepatocellular carcinoma transgenic mouse model," Molecular Therapy-Nucleic Acids, vol. 11, pp. 485493, 2018.

[40] G. Lou, L. Chen, C. Xia et al., "MiR-199a-modified exosomes from adipose tissue-derived mesenchymal stem cells improve hepatocellular carcinoma chemosensitivity through mTOR pathway," Journal of Experimental \& Clinical Cancer Research, vol. 39, no. 1, p. 4, 2020.

[41] D. Jiang, W. C. Cho, Z. Li et al., "MiR-758-3p suppresses proliferation, migration and invasion of hepatocellular carcinoma cells via targeting MDM2 and mTOR," Biomedicine \& Pharmacotherapy, vol. 96, pp. 535-544, 2017.

[42] D. Pollutri, C. Patrizi, S. Marinelli et al., "The epigenetically regulated miR-494 associates with stem-cell phenotype and induces sorafenib resistance in hepatocellular carcinoma," Cell Death \& Disease, vol. 9, no. 1, p. 4, 2018.

[43] H. Tang, R.-P. Li, P. Liang, Y.-L. Zhou, and G.-W. Wang, "miR-125a inhibits the migration and invasion of liver cancer cells via suppression of the PI3K/AKT/mTOR signaling pathway," Oncology Letters, vol. 10, no. 2, pp. 681-686, 2015.

[44] M. Yu, H. Xue, Y. Wang et al., "miR-345 inhibits tumor metastasis and EMT by targeting IRF1-mediated mTOR/ STAT3/AKT pathway in hepatocellular carcinoma," International Journal of Oncology, vol. 50, no. 3, pp. 975-983, 2017.

[45] Q. Song, H. Zhang, J. He et al., "Long non-coding RNA LINC00473 acts as a microRNA-29a-3p sponge to promote hepatocellular carcinoma development by activating Robo1- dependent PI3K/AKT/mTOR signaling pathway," Therapeutic Advances in Medical Oncology, vol. 12, 2020.

[46] X. Dong, "MicroRNA-9-5p downregulates Klf4 and influences the progression of hepatocellular carcinoma via the AKT signaling pathway," International Journal of Molecular Medicine, vol. 43, no. 3, pp. 1417-1429, 2019.

[47] Y. Chang, C. Zhou, L. Fan et al., "Upregulation of microRNA300 induces the proliferation of liver cancer by downregulating transcription factor FOXO1," Oncology reports, vol. 40, no. 6, pp. 3561-3572, 2018.

[48] S. Chang, "Long non-coding RNA XIST regulates PTEN expression by sponging miR-181a and promotes hepatocellular carcinoma progression," BMC Cancer, vol. 17, no. 1, p. 248, 2017.

[49] A. Sanchez-Mejias, J. Kwon, X. H. Chew et al., "A novel SOCS5/miR-18/miR-25 axis promotes tumorigenesis in liver cancer," International Journal of Cancer, vol. 144, no. 2, pp. 311-321, 2019.

[50] H.-C. Zhou, J.-H. Fang, L.-R. Shang et al., "MicroRNAs miR$125 \mathrm{~b}$ and miR-100 suppress metastasis of hepatocellular carcinoma by disrupting the formation of vessels that encapsulate tumour clusters," The Journal of Pathology, vol. 240, no. 4, pp. 450-460, 2016.

[51] Z. Dong, R. Qi, X. Guo et al., "MiR-223 modulates hepatocellular carcinoma cell proliferation through promoting apoptosis via the Rab1-mediated mTOR activation," Biochemical and Biophysical Research Communications, vol. 483, no. 1, pp. 630-637, 2017.

[52] S. Djebali, C. A Davis, A Merkel et al., "Landscape of transcription in human cells," Nature, vol. 489, no. 7414, pp. 101-108, 2012.

[53] Y. Huang, "The novel regulatory role of lncRNA-miRNAmRNA axis in cardiovascular diseases," Journal of Cellular and Molecular Medicine, vol. 22, no. 12, pp. 5768-5775, 2018.

[54] W.-X. Peng, P. Koirala, and Y.-Y. Mo, "LncRNA-mediated regulation of cell signaling in cancer," Oncogene, vol. 36, no. 41 , pp. 5661-5667, 2017.

[55] A. Bhan, M. Soleimani, and S. S. Mandal, "Long noncoding RNA and cancer: a new paradigm," Cancer Research, vol. 77, no. 15, pp. 3965-3981, 2017.

[56] L. Ma, V. B. Bajic, and Z. Zhang, "On the classification of long non-coding RNAs,” RNA Biology, vol. 10, no. 6, pp. 925-933, 2013.

[57] L. J. Lim, S. Y. S. Wong, F. Huang et al., "Roles and regulation of long noncoding RNAs in hepatocellular carcinoma," Cancer Research, vol. 79, no. 20, pp. 5131-5139, 2019.

[58] Y. Wu, Y. Zhang, X. Qin, H. Geng, D. Zuo, and Q. Zhao, "PI3K/AKT/mTOR pathway-related long non-coding RNAs: roles and mechanisms in hepatocellular carcinoma," Pharmacological Research, vol. 160, p. 105195, 2020.

[59] L. Wei, X. Yang, L. Lv et al., "The emerging role of microRNAs and long noncoding RNAs in drug resistance of hepatocellular carcinoma," Molecular Cancer, vol. 18, no. 1, p. 147, 2019.

[60] J. Li, D. S Zhai, Q Huang, H. L Chen, Z Zhang, and Q. F Tan, "LncRNA DCST1-AS1 accelerates the proliferation, metastasis and autophagy of hepatocellular carcinoma cell by AKT/ mTOR signaling pathways," European Review for Medical and Pharmacological Sciences, vol. 23, no. 14, pp. 6091-6104, 2019.

[61] W. Chen, J. You, Q. Zheng, and Y. Zhu, "Downregulation of lncRNA OGFRP1 inhibits hepatocellular carcinoma progression by $\mathrm{AKT} / \mathrm{mTOR}$ and $\mathrm{Wnt} / \beta$-catenin signaling pathways," Cancer Management and Research, vol. 10, pp. 1817-1826, 2018. 
[62] X. Guo and Y. Wang, "LncRNA TMPO-AS1 promotes hepatocellular carcinoma cell proliferation, migration and invasion through sponging miR-329-3p to stimulate FOXK1mediated AKT/mTOR signaling pathway," Cancer Medicine, vol. 9, 2020.

[63] N. Peng, J. He, J. Li et al., "Long noncoding RNA MALAT1 inhibits the apoptosis and autophagy of hepatocellular carcinoma cell by targeting the microRNA-146a/PI3K/Akt/ mTOR axis," Cancer Cell International, vol. 20, p. 165, 2020.

[64] P. Malakar, I. Stein, A. Saragovi et al., "Long noncoding RNA MALAT1 regulates cancer glucose metabolism by enhancing mTOR-mediated translation of TCF7L2," Cancer Research, vol. 79, no. 10, pp. 2480-2493, 2019.

[65] B. Ye, B. Hu, Z. Zheng, R. Zheng, and Y. Shi, "The long noncoding RNA AK023948 enhances tumor progression in hepatocellular carcinoma," Experimental and Therapeutic Medicine, vol. 14, no. 4, pp. 3658-3664, 2017.

[66] H. Wei, J. Hu, J. Pu et al., "Long noncoding RNA HAGLROS promotes cell proliferation, inhibits apoptosis and enhances autophagy via regulating miR-5095/ATG12 axis in hepatocellular carcinoma cells," International Immunopharmacology, vol. 73, pp. 72-80, 2019.

[67] Y. Huang, B. Xiang, Y. Liu, Y. Wang, and H. Kan, "LncRNA CDKN2B-AS1 promotes tumor growth and metastasis of human hepatocellular carcinoma by targeting let-7c-5p/ NAP1L1 axis," Cancer Letters, vol. 437, pp. 56-66, 2018.

[68] J. H. Zhong, X. Xiang, Y. Y. Wang et al., "The lncRNA SNHG16 affects prognosis in hepatocellular carcinoma by regulating p62 expression," Journal of Cellular Physiology, vol. 235, no. 2, pp. 1090-1102, 2020.

[69] Y. Han, M. Chen, A. Wang, and X. Fan, "STAT3-induced upregulation of lncRNA CASC11 promotes the cell migration, invasion and epithelial-mesenchymal transition in hepatocellular carcinoma by epigenetically silencing PTEN and activating PI3K/AKT signaling pathway," Biochemical and Biophysical Research Communications, vol. 508, no. 2, pp. $472-479,2019$.

[70] K. Han, C. Li, X. Zhang, and L. Shang, "DUXAP10 inhibition attenuates the proliferation and metastasis of hepatocellular carcinoma cells by regulation of the $\mathrm{Wnt} / \beta$-catenin and PI3K/ Akt signaling pathways," Bioscience Reports, vol. 39, no. 5, 2019.

[71] Z. Liu, H. Mo, L. Sun et al., "Long noncoding RNA PICSAR/ miR-588/EIF6 axis regulates tumorigenesis of hepatocellular carcinoma by activating PI3K/AKT/mTOR signaling pathway," Cancer Science, vol. 111, no. 11, pp. 4118-4128, 2020.

[72] G. Shu, H. Su, Z. Wang et al., "LINC00680 enhances hepatocellular carcinoma stemness behavior and chemoresistance by sponging miR-568 to upregulate AKT3," Journal of Experimental \& Clinical Cancer Research, vol. 40, no. 1, p. 45, 2021.

[73] X. Xin, M. Wu, Q. Meng et al., "Long noncoding RNA HULC accelerates liver cancer by inhibiting PTEN via autophagy cooperation to miR15a," Molecular Cancer, vol. 17, no. 1, p. 94, 2018.

[74] Y. Ma, D. Cao, G. Li, J. Hu, X. Liu, and J. Liu, "Silence of lncRNA HEIH suppressed liver cancer cell growth and metastasis through miR-199a-3p/mTOR axis," Journal of Cellular Biochemistry, vol. 120, no. 10, pp. 17757-17766, 2019.

[75] S. Wei, Q. Fan, L. Yang et al., "Promotion of glycolysis by HOTAIR through GLUT1 upregulation via mTOR signaling," Oncology Reports, vol. 38, no. 3, pp. 1902-1908, 2017.

[76] W. Li, Q. Fu, W. Man, H. Guo, and P. Yang, "LncRNA OR3A4 participates in the angiogenesis of hepatocellular carcinoma through modulating AGGF1/akt/mTOR pathway," European Journal of Pharmacology, vol. 849, pp. 106-114, 2019.

[77] F. Hong, Y. Gao, Y. Li, L. Zheng, F. Xu, and X. Li, "Inhibition of HIF1A-AS1 promoted starvation-induced hepatocellular carcinoma cell apoptosis by reducing HIF- $1 \alpha /$ mTOR-mediated autophagy," World Journal of Surgical Oncology, vol. 18, no. 1, p. 113, 2020.

[78] S. Meng, H. Zhou, Z. Feng et al., "CircRNA: functions and properties of a novel potential biomarker for cancer," Molecular Cancer, vol. 16, no. 1, p. 94, 2017.

[79] H. L. Sanger, G. Klotz, D. Riesner, H. J. Gross, and A. K. Kleinschmidt, "Viroids are single-stranded covalently closed circular RNA molecules existing as highly base-paired rod-like structures," Proceedings of the National Academy of Sciences, vol. 73, no. 11, pp. 3852-3856, 1976.

[80] Y. Zhao, "Deficiency in the ubiquitin conjugating enzyme UBE2A in Alzheimer's disease (AD) is linked to deficits in a natural circular miRNA-7 sponge (circRNA; ciRS-7)," Genes (Basel), vol. 7, no. 12, 2016.

[81] H. Xu, S. Guo, W. Li, and P. Yu, "The circular RNA Cdrlas, via miR-7 and its targets, regulates insulin transcription and secretion in islet cells," Scientific Reports, vol. 5, p. 12453, 2015.

[82] L. M. Holdt, A. Stahringer, K. Sass et al., "Circular non-coding RNA ANRIL modulates ribosomal RNA maturation and atherosclerosis in humans," Nature Communications, vol. 7, p. 12429, 2016.

[83] M. Zhang, N. Huang, X. Yang et al., "A novel protein encoded by the circular form of the SHPRH gene suppresses glioma tumorigenesis," Oncogene, vol. 37, no. 13, pp. 1805-1814, 2018.

[84] J. Guarnerio, M. Bezzi, J. C. Jeong et al., "Oncogenic role of fusion-circRNAs derived from cancer-associated chromosomal translocations," Cell, vol. 165, no. 2, pp. 289-302, 2016.

[85] J. Yu, Q.-g. Xu, Z.-g. Wang et al., "Circular RNA cSMARCA5 inhibits growth and metastasis in hepatocellular carcinoma," Journal of Hepatology, vol. 68, no. 6, pp. 1214-1227, 2018.

[86] X. Zhang, S. Wang, H. Wang et al., "Circular RNA circNRIP1 acts as a microRNA-149-5p sponge to promote gastric cancer progression via the AKT1/mTOR pathway," Molecular Cancer, vol. 18, no. 1, p. 20, 2019.

[87] X. Y. Huang, Z. L. Huang, P. B. Zhang et al., "CircRNA100338 is associated with mTOR signaling pathway and poor prognosis in hepatocellular carcinoma," Frontiers in Oncology, vol. 9, p. 392, 2019.

[88] X. H. Sun, Y.-T. Wang, G.-F. Li, N. Zhang, and L. Fan, "Serum-derived three-circRNA signature as a diagnostic biomarker for hepatocellular carcinoma," Cancer Cell International, vol. 20, p. 226, 2020.

[89] H. Zheng, T. Chen, C. Li et al., "A circular RNA hsa_circ_ 0079929 inhibits tumor growth in hepatocellular carcinoma," Cancer Management and Research, vol. 11, pp. 443-454, 2019.

[90] A. Koch, S. C. Joosten, Z. Feng et al., "Analysis of DNA methylation in cancer: location revisited," Nature Reviews Clinical Oncology, vol. 15, no. 7, pp. 459-466, 2018.

[91] X. Liu, X. Hu, Y. Kuang et al., "BCLB, methylated in hepatocellular carcinoma, is a starvation stress sensor that induces apoptosis and autophagy through the AMPK-mTOR signaling cascade," Cancer Letters, vol. 395, pp. 63-71, 2017.

[92] G. Almouzni and H. Cedar, "Maintenance of epigenetic information," Cold Spring Harbor Perspectives in Biology, vol. 8, no. 5, 2016.

[93] M. Berdasco and M. Esteller, "Aberrant epigenetic landscape in cancer: how cellular identity goes awry," Developmental Cell, vol. 19, no. 5, pp. 698-711, 2010. 
[94] Z. Y. Zhang, D. Hong, S. H. Nam et al., "SIRT1 regulates oncogenesis via a mutant p53-dependent pathway in hepatocellular carcinoma," Journal of Hepatology, vol. 62, no. 1, pp. 121-130, 2015.

[95] G. Wang, Y. Huang, F. Yang et al., "High expression of SMYD3 indicates poor survival outcome and promotes tumour progression through an IGF-1R/AKT/E2F-1 positive feedback loop in bladder cancer," Aging, vol. 12, no. 3, pp. 2030-2048, 2020.

[96] J. Makarević, N Tawanaie, E Juengel et al., "Cross-communication between histone $\mathrm{H} 3$ and $\mathrm{H} 4$ acetylation and AktmTOR signalling in prostate cancer cells," Journal of Cellular and Molecular Medicine, vol. 18, no. 7, pp. 1460-1466, 2014.

[97] D.-f. Sun, Y.-j. Zhang, X.-q. Tian, Y.-x. Chen, and J.-y. Fang, "Inhibition of mTOR signalling potentiates the effects of trichostatin A in human gastric cancer cell lines by promoting histone acetylation," Cell Biology International, vol. 38, no. 1, pp. 50-63, 2014.

[98] J. Masliah-Planchon, I. Bièche, J.-M. Guinebretière, F. Bourdeaut, and O. Delattre, "SWI/SNF chromatin remodeling and human malignancies," Annual Review of Pathology: Mechanisms of Disease, vol. 10, no. 1, pp. 145-171, 2015.

[99] Y. Zhou, Q. Xu, L. Tao et al., "Enhanced SMARCD1, a subunit of the SWI/SNF complex, promotes liver cancer growth through the mTOR pathway," Clinical Science, vol. 134, no. 12, pp. 1457-1472, 2020. 